RELATING FIBER CROSSING IN HARDI TO INTELLECTUAL FUNCTION

By

Iman Aganj, Neda Jahanshad, Christophe Lenglet, Arthur W. Toga, Katie L. McMahon,

Greig I. de Zubicaray, Margaret J. Wright, Nicholas G. Martin, Guillermo Sapiro

and

Paul M. Thompson

IMA Preprint Series \# 2298

(February 2010)

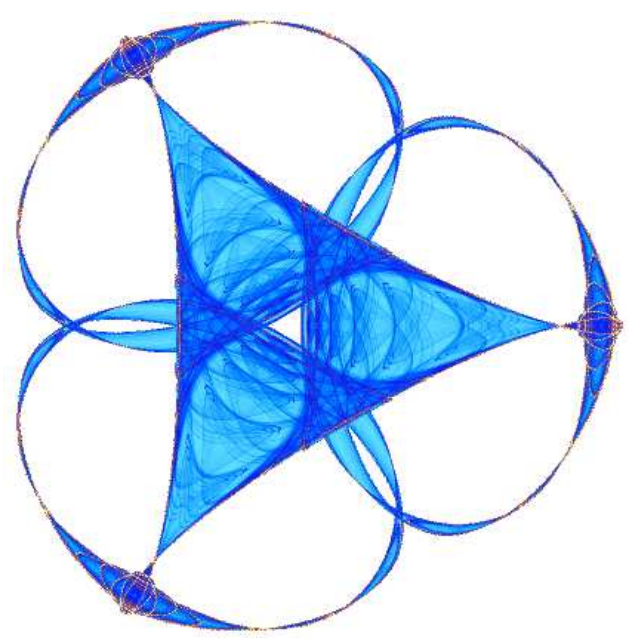

INSTITUTE FOR MATHEMATICS AND ITS APPLICATIONS

UNIVERSITY OF MINNESOTA 400 Lind Hall

207 Church Street S.E.

Minneapolis, Minnesota 55455-0436

Phone: 612-624-6066 Fax: 612-626-7370

URL: http://www.ima.umn.edu 


\title{
Relating Fiber Crossing in HARDI to Intellectual Function
}

\author{
Iman Aganj, ${ }^{1}$ Neda Jahanshad,${ }^{2}$ Christophe Lenglet, ${ }^{1,3}$ Arthur W. Toga, ${ }^{2}$ Katie L. McMahon, ${ }^{4}$ \\ Greig I. de Zubicaray, ${ }^{4}$ Margaret J. Wright, ${ }^{5}$ Nicholas G. Martin, ${ }^{5}$ \\ Guillermo Sapiro, ${ }^{1}$ and Paul M. Thompson ${ }^{2}$ \\ ${ }^{1}$ Dept. Electrical and Computer Engineering, University of Minnesota, MN, USA \\ ${ }^{2}$ Laboratory of Neuro Imaging, Dept. of Neurology, UCLA School of Medicine, CA, USA \\ ${ }^{3}$ Center for Magnetic Resonance Research, University of Minnesota, MN, USA \\ ${ }^{4}$ University of Queensland, Functional MRI Laboratory, Centre for MR, Brisbane, Australia \\ ${ }^{5}$ Queensland Institute of Medical Research, Brisbane, Australia
}

Introduction: Localizing brain structures and pathways associated with intellectual performance and cognition may clarify the networks involved, their development, and their dysfunction in disease. Here we introduce a measure of fiber crossing computed from high angular resolution diffusion images (HARDI), with the goal of better characterizing complex white matter microstructure. We aimed to detect white matter features associated with intellectual performance, previously missed due to limitations of the single tensor diffusion model.

Methods: High magnetic field (4T) diffusion-weighted (94 gradients, 11 non-sensitized) MR images were acquired from 293 right-handed healthy young adult twins and their siblings (23.9 \pm 1.9 years old; 112 monozygotic subjects, 153 dizygotic, and 28 siblings). Verbal, performance, and full-scale intelligence quotients were also measured (VIQ: $114.7 \pm 15.2$; PIQ: $114.2 \pm 11.9$; FIQ: $111.7 \pm 10.4$ ). Images were corrected for motion and eddy current distortions. Each subject's average b0 image was aligned to a common subject specific template using a nine-parameter affine transformation, and gradient directions were corrected accordingly. Orientation distribution functions (ODFs) in constant solid angle were computed as described in [1] from both the diffusion tensors [2] and q-balls [3]. We also created a mean map of the q-ball ODFs, averaged across all subjects. We computed the geodesic distance [4,5] between the two ODFs (from the fitted tensor and q-ball) at each voxel for each subject. Both the tensor and q-ball ODF models can correctly reconstruct isotropic and single-fiber geometries. However, only the q-ball ODF can correctly resolve fiber crossings, so the mentioned tensor-q-ball ODF distance (TQOd) is a reasonable measure of fiber crossing. Each individual's fractional anisotropy (FA) maps were elastically registered to a common FA template including only high FA regions, to improve white matter registration. Individual deformation fields were then applied to the TQOd to identically align each subject's FA and TQOd maps. Using a linear random effects regression analysis to account for familial relations in the twin samples, voxelwise FA and TQOd maps were each regressed against each intelligence score.

Results: Figures 1 and 2 show the mean q-ball ODF field overlaid on the mean FA and mean TQOd maps. The mean TQOd clearly identifies known crossings, e.g. where the corpus callosum, corona radiata and internal capsule all intersect. Known isotropic, single-fiber anisotropic, and fiber crossing regions were identifiable, respectively, as regions with low FA/low TQOd, high FA/low TQOd, and low FA/high TQOd. After correcting for multiple comparisons using the false discovery rate (FDR) method, performance IQ - which is thought to be related to neuronal processing speed - was associated with high anisotropy in several regions, as expected [6]. TQOd correlations with PIQ were also significant in 
regions of low FA located in the caudate head, where partial voluming occurs between the internal capsule and striatum. Here, fiber crossing and the presence of multiple tissue types per voxel prevent tensors from fitting correctly, but q-ball reconstruction is more accurate. Figure $\mathbf{3}$ shows voxels that survived FDR correction, with uncorrected p-values of $\mathrm{p}<3.2 \times 10^{-5}$ for TQOd.

Conclusions: We introduced a new measure to identify white matter fiber crossings in HARDI; this measure was correlated with performance IQ in brain regions where correlation with FA was not detected. This highlights the importance of higher-order diffusion modeling to better localize fiber pathways relevant for cognition.

Acknowledgments: Supported by NIH (P41 RR008079, P30 NS057091, R01 HD050735, R01 EB007813, R01 MH060662, R01 EB008432, CON000000015793-3014, NLM T15 LM07356), ONR, NGA, NSF, DARPA, ARO, and NHMRC (Australia).

\section{References:}

[1] Aganj, I., Lenglet, C., Sapiro, G., Yacoub, E., Ugurbil, K. and Harel, N. (2009) 'Reconstruction of the orientation distribution function in single and multiple shell q-ball imaging within constant solid angle,' Magnetic Resonance in Medicine, in press.

[2] Basser, P.J., Mattiello, J. and LeBihan, D. (1994) 'Estimation of the effective self-diffusion tensor from the NMR spin echo,' J Magn Reson B., vol. 103, no. 3, p. 247-254.

[3] Tuch, D.S. (2004) 'Q-ball imaging,' Magnetic Resonance in Medicine, vol. 52, no. 6, p. 1358-1372.

[4] Goh, A., Lenglet, C., Thompson, P. and Vidal, R. (2009) 'A nonparametric Riemannian framework for processing High Angular Resolution Diffusion Images (HARDI), IEEE Conference on Computer Vision and Pattern Recognition, p. 2496-2503.

[5] Cheng, J., Ghosh, A., Jiang, T. and Deriche, R. (2009) 'A Riemannian framework for orientation distribution function computing,' Proc. of $12^{\text {th }}$ International Conference on Medical Image Computing and Computer Assisted Intervention, p. 911-918.

[6] Chiang, M.C. et al. (2009) 'Genetics of Brain Fiber Architecture and Intellectual Performance,' Journal of Neuroscience, vol. 29, no. 7, p. 2212-2224. 


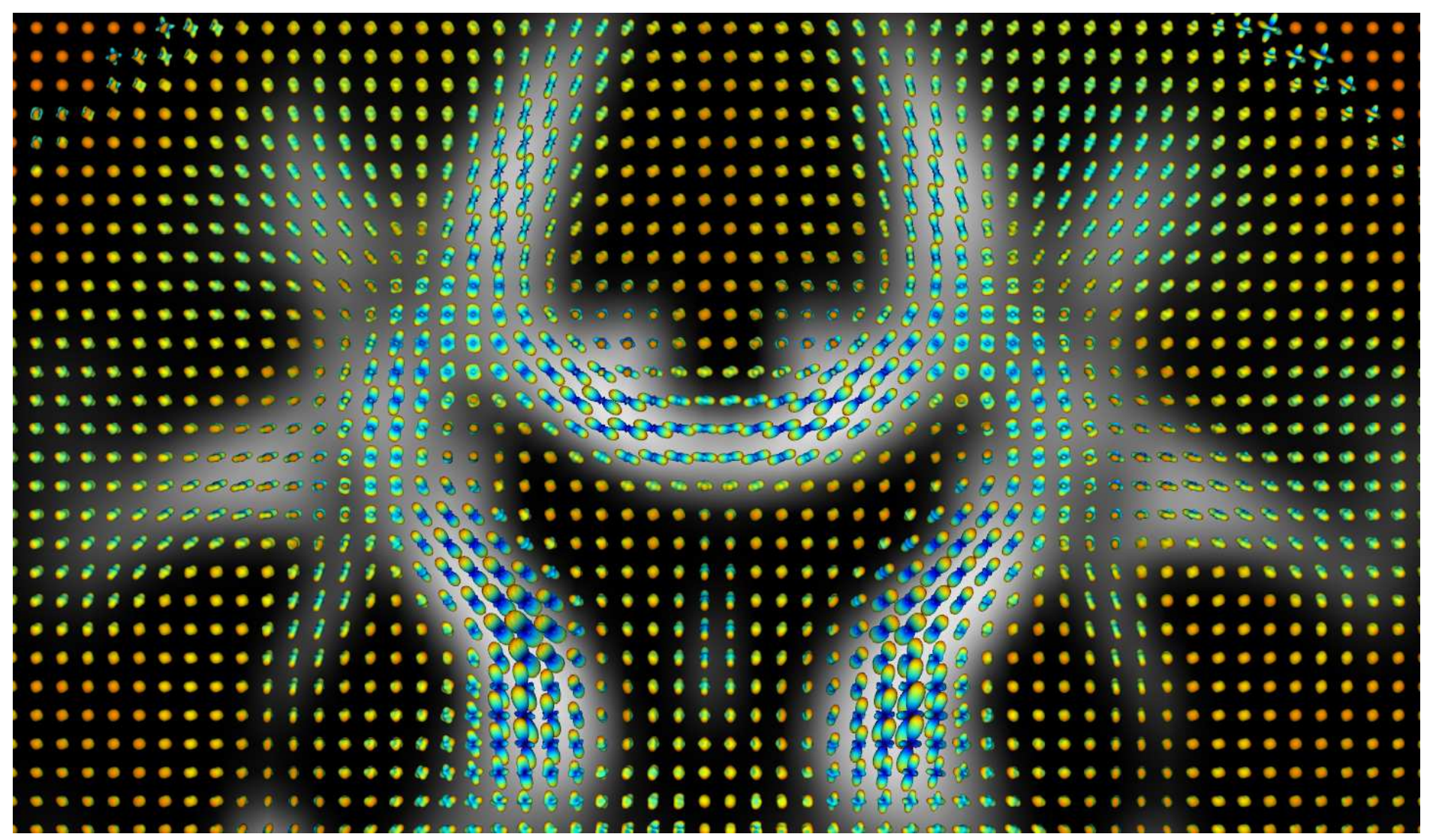

Fig. 1 


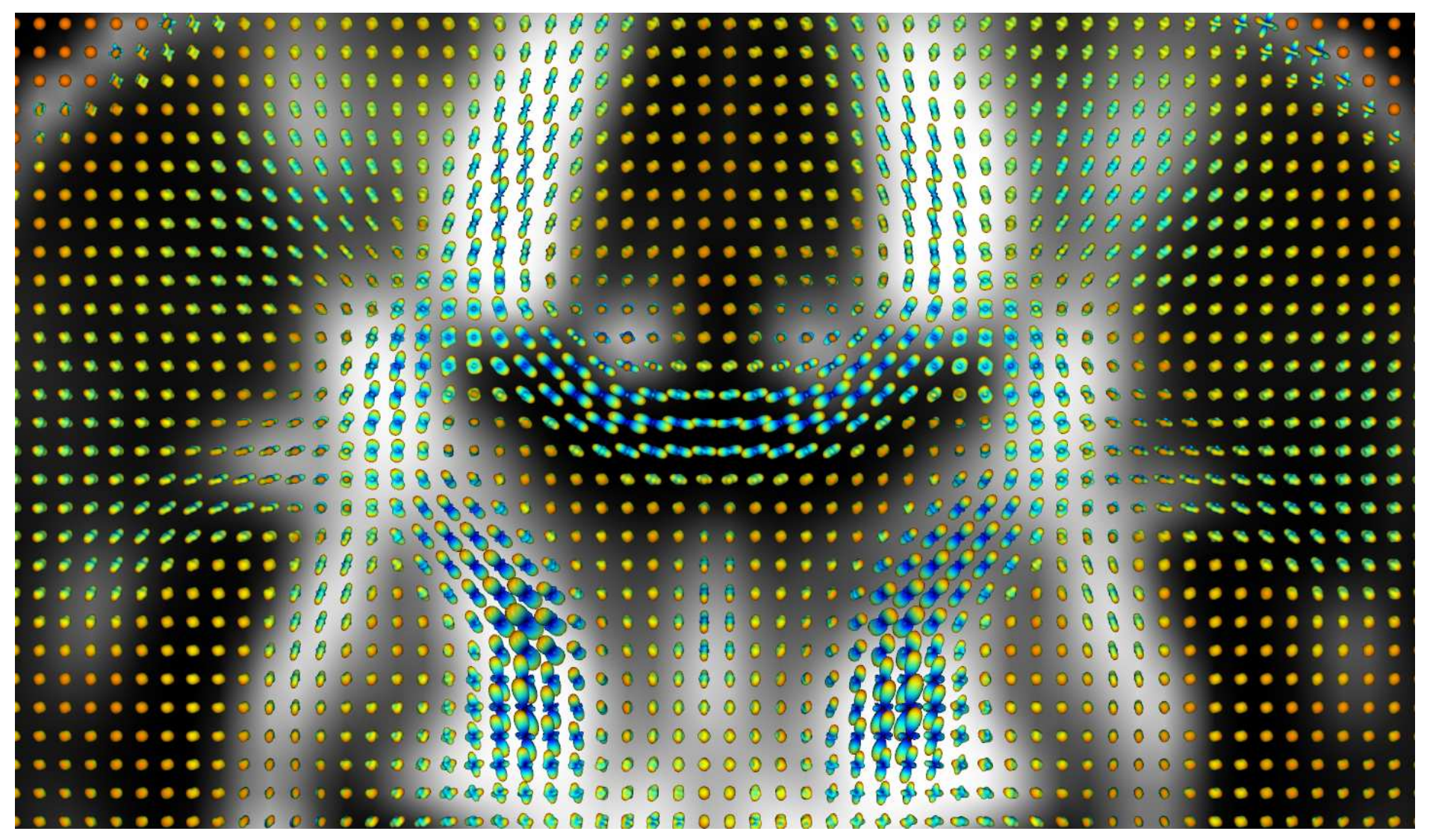

Fig. 2
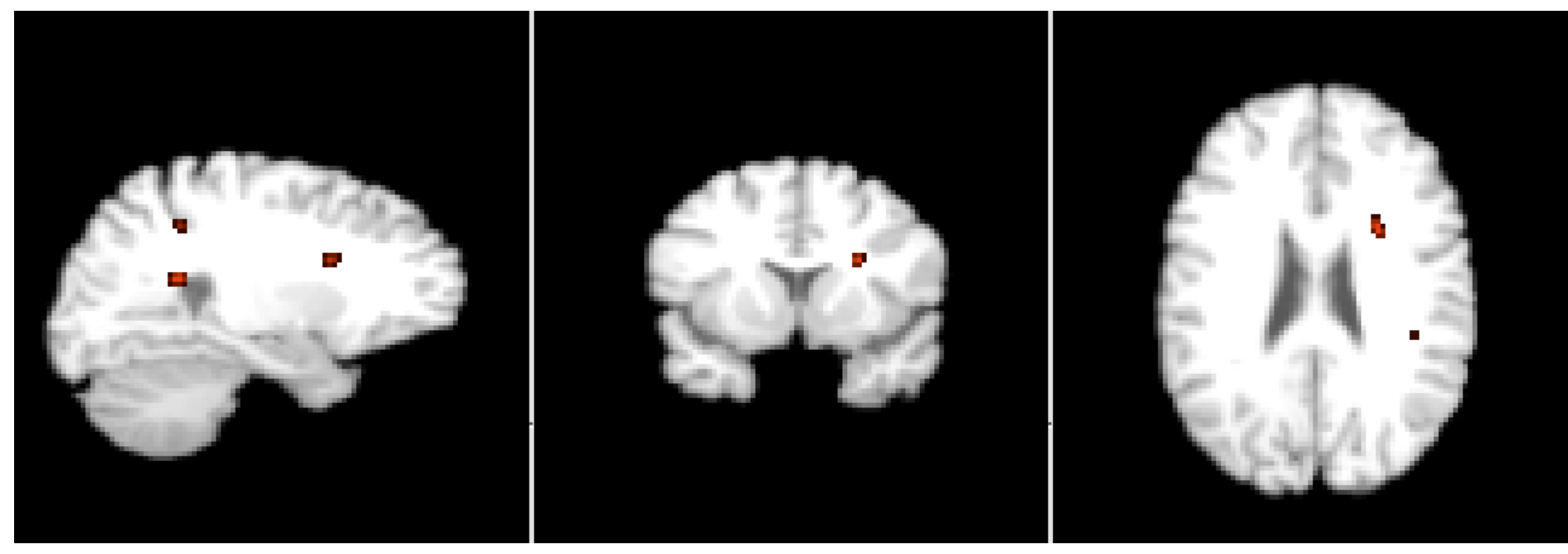

Fig. 3 